\title{
SEC-SAXS on an in-house laboratory instrument
}

\author{
Soren Skou ${ }^{\mathrm{a}}$, Saskia Bucciarelli ${ }^{\mathrm{b}}$, Soren R. Midtgaard ${ }^{\mathrm{c}}$, Martin N. Pedersen ${ }^{\mathrm{c}}$, Lise Arleth ${ }^{\mathrm{c}}$, \\ Bente Vestergaard ${ }^{\mathrm{b}}$
}

aXenocs Nordic, Hørsholm, 2970, Denmark, soren.skou@ saxslab.com

${ }^{\mathrm{b} C}$ Copenhagen University, Dept. of Drug Design and Pharmacology, 2100, Copenhagen, Denmark

${ }^{\mathrm{c} C}$ Copenhagen University, Niels Bohr Institute, 2100, Copenhagen, Denmark

Size-exclusion chromatography in combination with biological solution small angle X-ray scattering (SEC-SAXS) is a useful technique that enables structural analysis of challenging samples such as labile biological samples and complex mixtures and is used extensively on all state-of-the-art biological SAXS beamlines at synchrotrons today.

Here, we describe the successful setup of a laboratory-based SEC-SAXS system, schematically shown in Figure 1. We present data from various proteins covering a broad range of molecular weights to demonstrate that high quality data can be obtained using small sample volumes at moderate concentrations, even from minor solution components, such as protein dimers. A pitfall traditionally encountered with SEC-SAXS is peak broadening and sample dilution, hampering accurate concentration determination of the measured samples.

Here we show that UV absorption measurements directly on the SAXS exposure cell circumvent this issue and ensure accurate concentration determination, crucial for direct molecular weight determination from the scattering data. Absence of radiation damage, a problem typically encountered with synchrotron SEC-SAXS, allows the fractionation of samples after SEC-SAXS for further analysis using complementary biostructural techniques. Determination of the exact chromatogram on the SAXS exposure volume through direct UV measurements allows accurate correlation of the obtained results with the SEC-SAXS results. The ability to perform SEC-SAXS in-house for a wide range of samples thus allows more samples to be studied without the need for synchrotron beamtime [1].

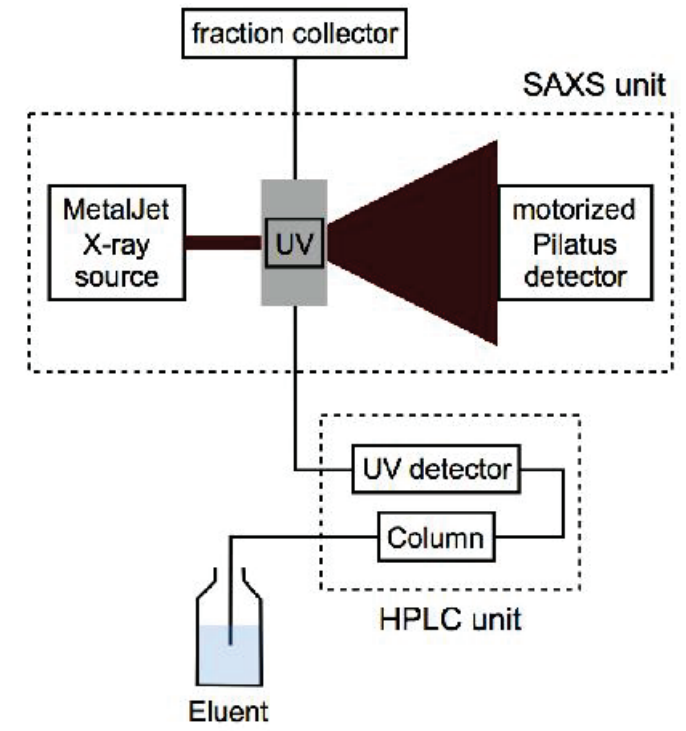

Fig. 1. SEC-SAXS setup schematic

$\underline{\text { References }}$

[1] Bucciarelli, S. et al. (2018). J. Appl. Cryst, 51(6), 1623-163 